UCRL-ID-127520

\title{
Report on Measurements at Ohio University to Estimate Backgrounds for Neutron Radiography in the 10-14 MeV Region
}

\author{
F. S Dietrich \\ J. M. Hall
}

May 10, 1997

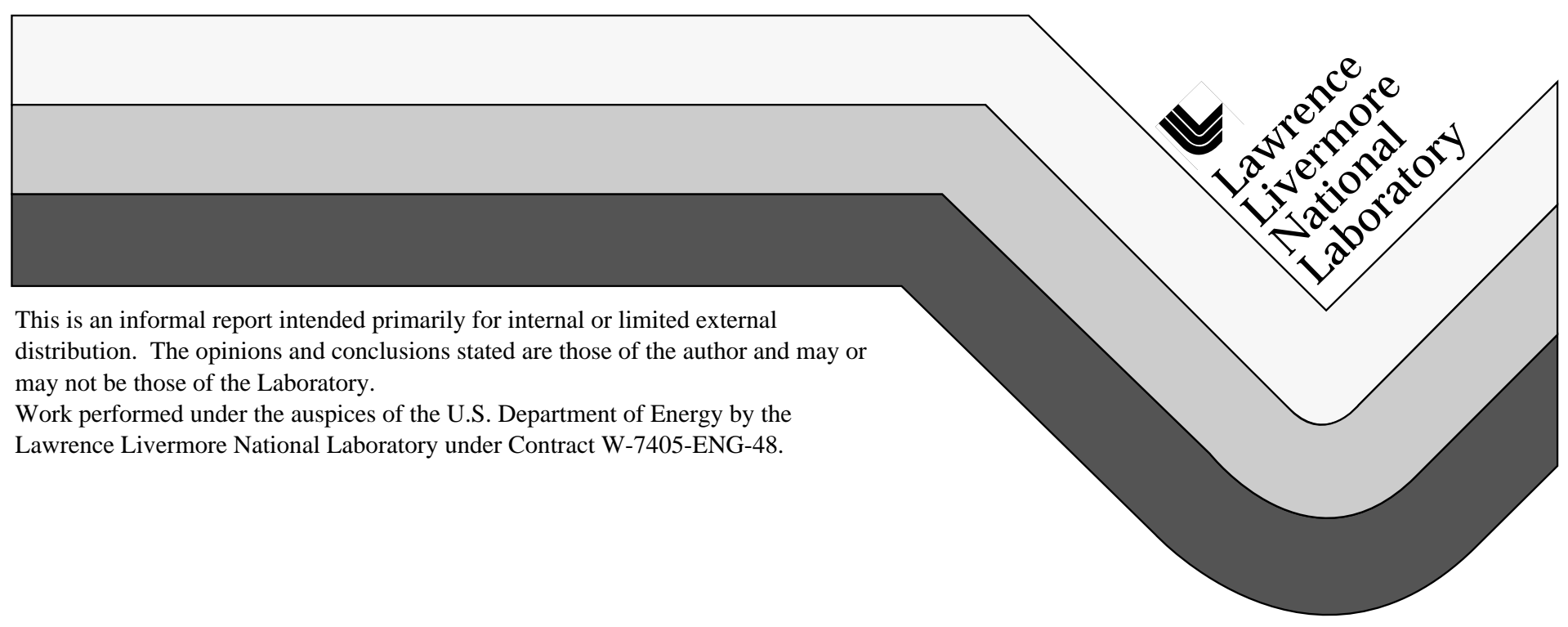




\section{DISCLAIMER}

This document was prepared as an account of work sponsored by an agency of the United States Government. Neither the United States Government nor the University of California nor any of their employees, makes any warranty, express or implied, or assumes any legal liability or responsibility for the accuracy, completeness, or usefulness of any information, apparatus, product, or process disclosed, or represents that its use would not infringe privately owned rights. Reference herein to any specific commercial product, process, or service by trade name, trademark, manufacturer, or otherwise, does not necessarily constitute or imply its endorsement, recommendation, or favoring by the United States Government or the University of California. The views and opinions of authors expressed herein do not necessarily state or reflect those of the United States Government or the University of California, and shall not be used for advertising or product endorsement purposes.

This report has been reproduced directly from the best available copy.

Available to DOE and DOE contractors from the Office of Scientific and Technical Information

P.O. Box 62, Oak Ridge, TN 37831

Prices available from (615) 576-8401, FTS 626-8401

Available to the public from the

National Technical Information Service

U.S. Department of Commerce 5285 Port Royal Rd.,

Springfield, VA 22161 


\title{
Report on Measurements at Ohio University to Estimate Backgrounds for Neutron Radiography in the 10-14 MeV Region
}

\author{
Frank S. Dietrich and James M. Hall \\ Lawrence Livermore National Laboratory
}

May 10, 1997

\section{Motivation}

In evaluating the feasibility of neutron radiography and tomography in the 10-14 $\mathrm{MeV}$ region, it is important to estimate the radiation backgrounds that could potentially interfere with the measurements. In this context, backgrounds refer to all counts in the detector other than those due to neutrons transmitted through the sample without scattering. There are two principal sources of backgrounds: (1) neutrons and gammas resulting from incident neutrons interacting in the sample, and (2) events in the detector arising from neutrons scattering in the accelerator vault and collimation system, together with natural and induced activation. Counts due to these backgrounds are spread fairly uniformly across the detector, and therefore do not compromise the ability to identify small features in the sample on the millimeter scale in a tomographic reconstruction; however, they do increase the neutron dose required to achieve sufficient statistical accuracy to reveal features of interest. Backgrounds are generally considered to be tolerable if their count rates are less than or comparable to the rates from the transmitted (uncollided) beam. If they are significantly above this level, they are a potentially serious problem.

Understanding radiation backgrounds is thus critically important in determining the required source strength and running time. The backgrounds must be characterized by their energy, radiation type (neutron or gamma), and their timing relative to emission time at the source. These properties may have a profound effect on the design of the source and detector (e.g., whether a pulsing-and-timing technique is necessary to reduce backgrounds, and whether a simple plastic-scintillator based integrating detector will suffice).

In the geometry that we have chosen to study, the sample is located approximately two meters from the neutron source, and the detector (a plastic-scintillator neutron-imaging camera; Ref. 1) is located another two meters downstream. A thick shielding wall with a collimating channel approximately $30 \mathrm{~cm}$ in diameter is located between the sample and detector to reduce room-scattered backgrounds.

We have studied the first source of background ("internal" or "sample" scattering) in this geometry using the COG Monte Carlo radiation transport code, and have found that these backgrounds should be tolerable (the effect of internal scattering should, in fact, be minimized in a system geometry with 2:1 magnification). The second type of background ("external" or "room" scattering and activation) is more difficult to study with a simulation code because these backgrounds are dependent on specific details of a facility that are difficult to know a priori. We have therefore carried out a measurement of these backgrounds in an existing facility, the Ohio University Accelerator Laboratory (OUAL), whose layout closely resembles the system geometry we envisage using for neutron radiography. These measurements were carried out in February, 1996. 


\section{Experimental Setup and Procedures}

The neutron time-of-flight facility at OUAL has been used for about 15 years in a program of precision neutron elastic and inelastic scattering measurements (cf. Figure 1). For neutron scattering experiments at this facility, charged particles (usually deuterons) with energies up to $11 \mathrm{MeV}$ from a tandem electrostatic accelerator are directed into a gas cell containing deuterium or tritium. The gas cell is typically 2 to $5 \mathrm{~cm}$ long and the beam spot at the cell is approximately $2 \mathrm{~mm}$ diameter. Neutrons of energies up to $26 \mathrm{MeV}$ produced by $\mathrm{D}+\mathrm{D}$ or $\mathrm{D}+\mathrm{T}$ reactions in the gas cell impinge on a scattering sample 10 to 20 $\mathrm{cm}$ from the source, and the scattered neutrons are counted in a detector placed 6 to $30 \mathrm{~m}$ from the neutron source. The detector is located in a well-shielded cave separated from the neutron source by a $1.52-\mathrm{m}$ thick wall pierced by a neutron collimation channel approximately $30 \mathrm{~cm}$ diameter. The distance from source to wall is approximately $2.3 \mathrm{~m}$. The incident charged-particle beam is pulsed and bunched into bursts of less than $1 \mathrm{~ns}$ duration, which permits neutron energies to be determined by time of flight. In neutron scattering experiments, a thick shadow bar blocks neutrons that would pass directly from the source to the detector without scattering from the sample. The angle between the neutrons incident on the scattering sample and the detected neutrons is varied by changing the direction of the charged particle beam with a moveable magnet system (the "beam swinger"). A detailed description of this facility may be found in Ref. 2.

This facility provides an appropriate setup for making the measurements we need to characterize room-scattered and activation backgrounds for neutron radiography. For these measurements, we used $10 \mathrm{MeV}$ neutrons from the $\mathrm{D}+\mathrm{D}$ reaction. We placed the beam swinger so that neutrons produced from this reaction at zero degrees are directed toward the detector located in the shielded cave at $5.35 \mathrm{~m}$ from the source. The measurements were made by observing the response of the detector with and without a long steel shadow bar placed in the source vault on a direct line between the source and the detector. The shadow bar was $15.2 \mathrm{~cm}$ in diameter and $79.4 \mathrm{~cm}$ long, which blocked all but a negligible fraction of the direct neutrons. A neutron monitor placed near the source was used to normalize the results with the shadow bar in and out.

The detector we employed was a liquid scintillator (BC-503) $12.7 \mathrm{~cm}$ in diameter and $5.08 \mathrm{~cm}$ thick, viewed by a fast photomultiplier tube. The response of this detector for neutrons and gammas is similar to that of the plastic scintillator (BC-408) we expect to use in the imaging system for radiography. However, the liquid scintillator allows the separate identification of neutron and gamma events using the dependence of pulse shape on ionization density that is characteristic of this type of detector. The pulse-height scale was calibrated with gamma sources $\left({ }^{137} \mathrm{Cs}\right.$ and $\left.{ }^{60} \mathrm{Co}\right)$. All events in the detector above a threshold of approximately $50 \mathrm{keV}$ gamma energy were recorded.

Events in the detector were analyzed using a standard data acquisition system. For each event, the pulse height, the pulse-shape discrimination signal carrying the neutron-vs.gamma information, and the time with respect to the deuteron beam pulses were recorded. One-dimensional histograms of these quantities were generated, together with all combinations of two-dimensional histograms (e.g., counts as a function of pulse height and time). This information allows a sufficiently complete characterization of the pulse height, time, and neutron and gamma sensitivities to evaluate the feasibility of using a plastic scintillator in an integrating mode for radiography and tomography applications. 


\section{Results of the Measurements}

An example of the sort of detailed information that was obtained in this experiment is shown in Figure 2. This figure shows the counts in the liquid scintillator with the direct neutron beam (i.e., with no shadow bar) as a function of time and the pulse shape discrimination (PSD) signal. The time and PSD scales are arbitrary and decreasing time channels correspond to increasing time of the events with respect to the beam pulse associated with them. The heavy line at PSD channel 17 shows the approximate division between gamma events below this channel, and the neutron events above it. The events in the sharp peaks near time channel 30 correspond to $10 \mathrm{MeV}$ neutrons from the reaction $\mathrm{D}+\mathrm{D} \rightarrow{ }^{3} \mathrm{He}+\mathrm{n}$, while those in the island centered around PSD channel 30 and time channel 15 are lower energy neutrons from the breakup reaction $D+D \rightarrow p+p+n+n$. The sharp peak near time channel 44 corresponds to gammas produced in and near the neutron-production gas cell. Note that energetic neutrons may produce both a neutron and a gamma response in the scintillator, as evidenced by the two peaks seen in the vicinity of time channel 30 . The latter is due to gamma production by neutrons in and around the detector.

In runs done with the shadow bar in place, the neutron signal is almost entirely absent in the PSD spectra. The observed counts are thus predominantly gamma rays. Furthermore, the time spectra show that these gamma rays are uniform in time (i.e., not correlated with the beam pulses). These counts correspond to a combination of activation gammas, and gammas interacting with the detector following radiative capture of neutrons that have scattered enough to lose their time correlation with the beam pulses. We isolated the contribution from long-half-life activation by taking runs with the beam turned off in several time intervals (typically 5 minutes) following completion of a run with the beam on. It was found that approximately half of the detector response with the shadow bar in place (determined by integrating the pulse-height spectrum with a weighting by pulse height) is due to naturally-occurring radiation backgrounds and residual activation built up over the 15 -year life of the facility.

To obtain a measure of the backgrounds relevant for neutron radiography applications, we have computed integrals of the pulse height spectra with and without the shadow bar in place. With the shadow bar out, this integral was computed in a narrow time interval containing the $10 \mathrm{MeV}$ neutrons. With the shadow bar in place, the pulseheight integral was taken over the entire time spectrum. In both cases, the integrals were computed with a pulse height weighting. This weighting is appropriate for evaluating the performance of an integrating detector such as the one we are currently planning to use for radiography, since each event contributes to the observed signal proportionally to its pulse height.

In the tests described here, we found that the ratio of the integral with the shadow bar in place to that with no shadow bar was approximately 0.0018 . This ratio is similar to the transmission of $10 \mathrm{MeV}$ neutrons through the thickest part of the samples that are of interest to us for neutron radiography and tomography applications. Apart from its placement in the detector cave, the detector used in these tests was entirely unshielded. Since the current measurements have shown that gammas are the dominant component of the background, it is reasonable to assume that significant further reduction in the background can be achieved with suitable shielding of the detector. 


\section{Conclusions}

The results of this experiment indicate that room-scattering and residual activation backgrounds are low enough to allow the use of an integrating plastic-scintillator-based detector in radiographic applications. It appears that neither time gating nor neutron/gamma discrimination will be necessary to obtain satisfactory images. This results in a significant simplification of the requirements for both the neutron source and the detector; however, it is clear that the detector must be placed in a sufficiently well isolated detector cave, and attention must be paid to optimizing the shielding in the neighborhood of the detector. While these measurements were carried out with $10 \mathrm{MeV}$ neutrons from the $\mathrm{D}+\mathrm{D}$ reaction, it is likely that the results would be similar for $14 \mathrm{MeV}$ neutrons from a D+T source. We currently favor a D+D source for a practical facility, largely because there is no need for handling tritium with this source. Based on the results of the test experiment reported here, we are constructing prototype imaging detector based on a plastic scintillator and CCD camera. This detector will be tested at the Ohio facility during late FY97.

\section{Acknowledgments}

We would like to thank Professor Roger W. Finlay and the staff of the Ohio University Accelerator Laboratory for their assistance in carrying out the measurements. 


\section{References}

1. F. Dietrich, J. Hall, and C. Logan, LLNL Report UCRL-JC-124401, Nov., 1996.

2. R. W. Finlay, et al., Nuclear Instruments and Methods 198, 197 (1982).

\section{Collected Figures}
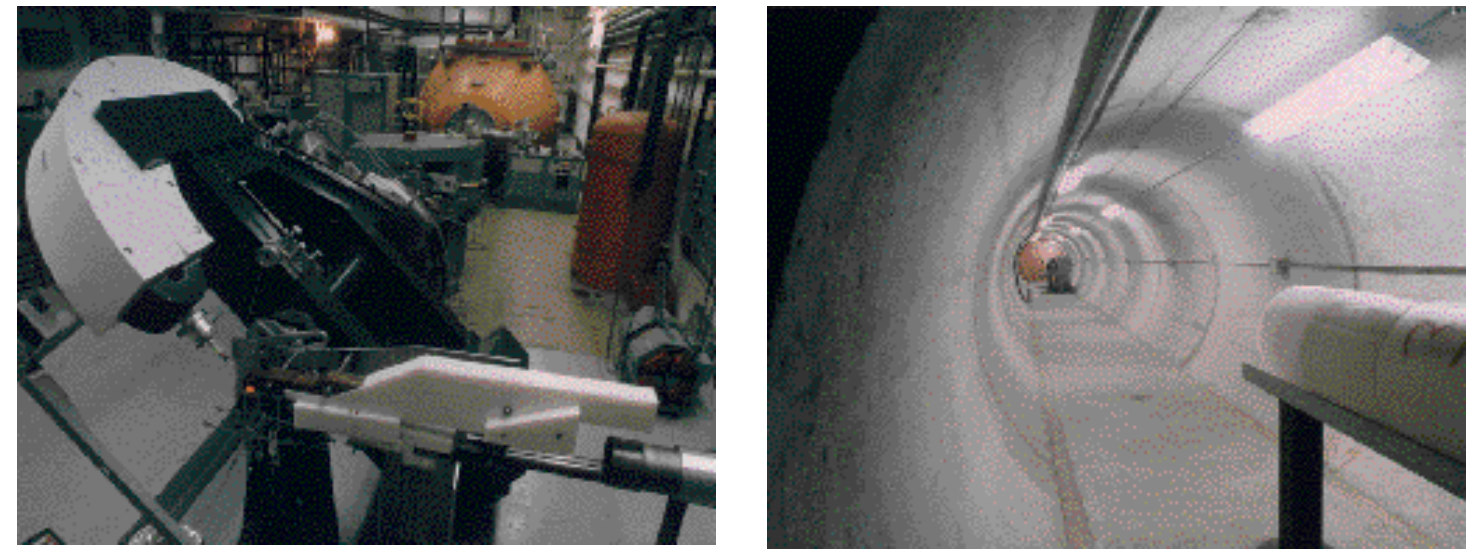

Figure 1. The Ohio University Accelerator Facility offers accelerator-driven DD and DT neutron sources and shielded detector caves which closely resemble the system geometry we envisage using for neutron radiography.

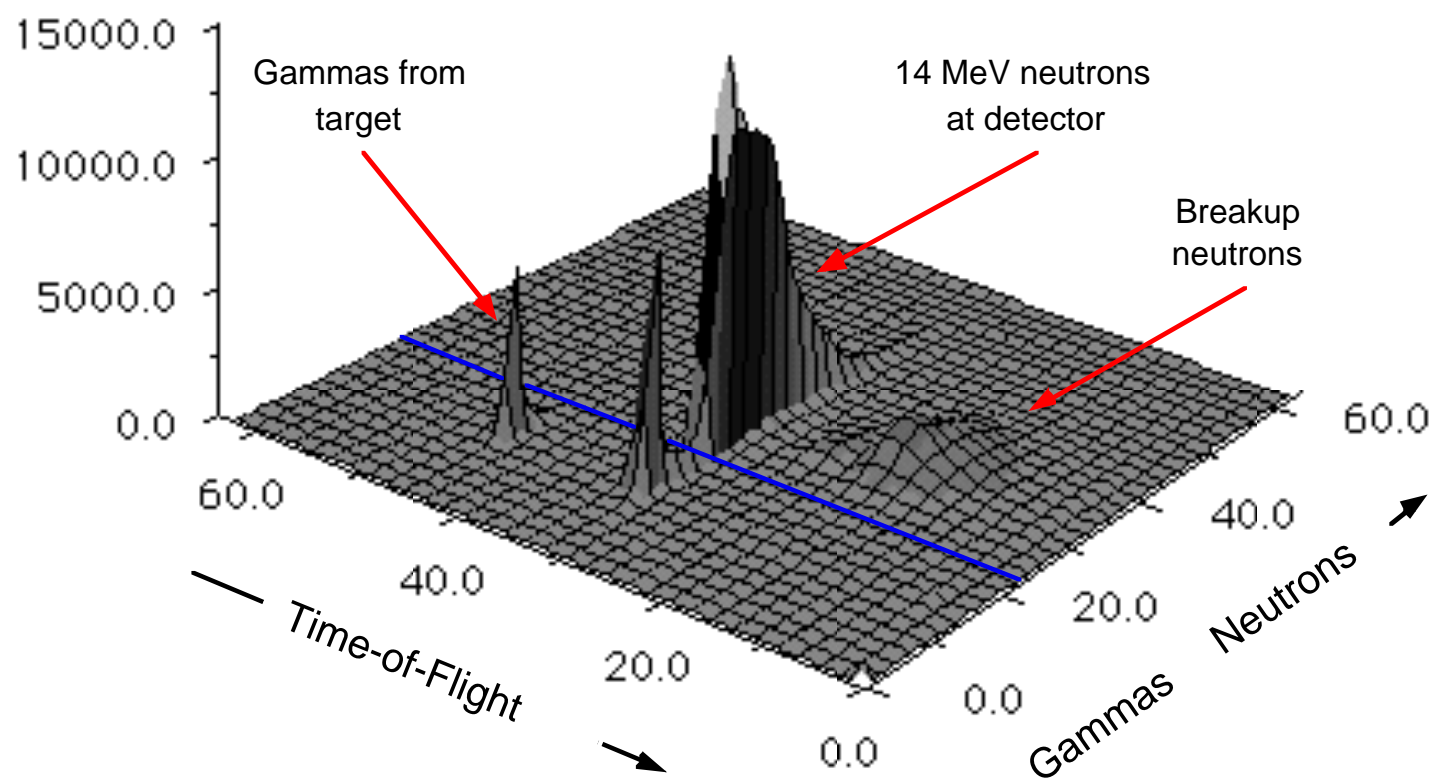

Figure 2. This plot shows the response of our liquid scintillator (BC-503) to $10 \mathrm{MeV}$ neutrons from the direct beam (no shadow bar). Observed counts are shown vs. time and pulse shape discrimination signals (arbitrary units). 